\title{
Genome-wide mapping for clinically relevant predictors of lamotrigine- and phenytoin-induced hypersensitivity reactions
}

\begin{abstract}
Aims: An association between carbamazepine-induced hypersensitivity and $H L A-A * 3101$ has been reported in populations of both European and Asian descent. We aimed to investigate $H L A-A * 3101$ and other common variants across the genome as markers for cutaneous adverse drug reactions (cADRs) attributed to lamotrigine and phenytoin. Materials \& methods: We recruited patients with lamotrigineinduced cADRs $(n=46)$ and patients with phenytoin-CADRs $(n=44)$ and the 1958 British birth cohort was used as a control $(n=1296)$. HLA-A*3101 was imputed from genome-wide association study data. We applied genome-wide association to study lamotrigine- and phenytoin-induced CADR, and total CADR cases combined. Results: Neither $H L A-A * 3101$ nor any other genetic marker significantly predicted lamotrigine- or phenytoin-induced CADRs. Conclusion: $H L A-A * 3101$ does not appear to be a predictor for lamotrigine- and phenytoin-induced CADRs in Europeans. Our genome-wide association study results do not support the existence of a clinically relevant common variant for the development of lamotrigine- or phenytoin-induced cADRs. As a predictive marker, HLA-A*3101 appears to be specific for carbamazepine-induced cADRs.
\end{abstract}

Original submitted 5 September 2011; Revision submitted 10 November 2011

\section{KEYWORDS: epilepsy GWAS HLA-A*3101 hypersensitivity lamotrigine phenytoin}

Carbamazepine (CBZ), lamotrigine (LTG) and phenytoin (PHT), among a class of antiepileptic drugs (AEDs) involved in sodium-channel modulation, are three of the leading first-line treatments for epilepsy. CBZ and PHT are among the earliest AEDs and are typically used to control partial seizures. LTG is well established as an effective drug for partial seizures, primaryand secondary, generalized tonic-clonic seizures, as well as for the treatment of Lennox-Gastaut syndrome. All of them have also been found to be useful in other conditions, such as bipolar disorder and trigeminal neuralgia.

Despite being highly effective, CBZ, LTG and PHT are associated with cutaneous adverse drug reactions (cADRs), which encompasses a mild maculopapular eruption (MPE), a more serious hypersensitivity syndrome (HSS) with liver and other internal organ involvement to the potentially fatal Stevens-Johnson syndrome (SJS) and/or toxic epidermal necrolysis (TEN). The highest rate of AED-related cADRs occurs with CBZ, LTG and PHT, each of them are structurally related through an aromatic ring, yet each are targeted towards different seizure profiles [1].

CBZ has been the focus of numerous genetic studies, especially since the discovery of the genetic marker $H L A-B^{*} 1502$ as a strong predictor for CBZ-induced SJS/TEN in subjects of Han Chinese ancestry [2]. Studies by the same group have also shown that the HLA$B^{*} 1502$ association with SJS/TEN extends to PHT and oxcarbazepine (OXC) [3]. Further research has illustrated that $H L A-B^{*} 1502$ also has a predictive ability for individuals of Malaysian, Indian, Thai and other Asian ethnicity [4-6]. The extent to which LTG-induced SJS/TEN associates with $H L A-B^{*} 1502$ remains to be elucidated and requires further investigation. As noted by Shi and colleagues, of the seven reported cases of LTG-induced SJS/TEN, three were positive for $H L A-B^{*} 1502$ [7]. To date, evidence from studies in Thai and Han Chinese populations suggests that there is no such association with the milder MPE phenotype triggered by either PHT or LTG $[5,7,8]$. However, a recent study has indicated that $H L A-B^{*} 1502$ may have predictive value for $\mathrm{OXC}$-induced MPE [9]. Although the authors only studied nine OXC-induced MPE cases, four of these were positive for $H L A-B^{*} 1502$, a significant observation when compared with population controls. These observations are particularly interesting given the close structural homology between OXC and CBZ.

However, the genetic association with $H L A-B^{*} 1502$ appears to be limited to Asian
Mark McCormack ${ }^{1}$,

Thomas J Urban²,

Kevin V Shianna², Nicole

Walley², Massimo

Pandolfo ${ }^{3}$, Chantal

Depondt ${ }^{3}$, Elijah Chaila ${ }^{4}$

Gerard D O'Conner ${ }^{4}$,

Dalia Kasperavičiüté ${ }^{5}$,

Rodney A Radtke ${ }^{2}$, Erin L

Heinzen², Sanjay M

Sisodiya ${ }^{5}$, Norman

Delanty ${ }^{1,4}$ \& Gianpiero L

Cavalleri*1

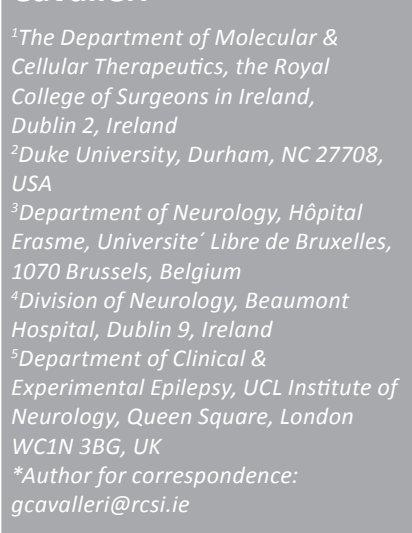

Future $\because$ Medicine 
populations. Studies in subjects of European ancestry have indicated that $H L A-B^{*} 1502$ is not present in CBZ-SJS patients of European descent $[10,11]$. This is not surprising considering the decreasing frequency of the $H L A-B^{*} 1502$ allele from a high frequency of $6 \%$ in Asia to virtually absent in Europe [12].

A 2006 study by Hung and colleagues reported an association between CBZ-induced MPE with $H L A-A^{*} 3101$ among patients of Han Chinese descent [13]. Using a genomewide approach, we identified $H L A-A^{*} 3101$ as a clinically relevant predictor for the full spectrum of CBZ-induced hypersensitivity reactions in Europeans, while a separate study by Ozeki and colleagues reported a significant association with $H L A-A^{*} 3101$ and CBZ-induced cADRs in a Japanese population [14,15]. The relationship between $H L A-A^{*} 3101$ and CBZ-induced HSS has now also been reported in patients of Korean descent [16].

Here we set out to investigate whether $H L A-A^{*} 3101$ or any other common genetic variant identified through genome-wide association studies (GWAS) may act as a predisposing factor for two of the other common AEDs known to cause cADRs, namely LTG and PHT.

\section{Materials \& methods Case subjects}

We obtained approval for this study from the local research ethics committees of each site. Written, informed consent was obtained from all subjects prior to participation. Patients were recruited through centers affiliated with the EPIGEN Consortium; namely Duke University (NC, USA), University College London (London, UK), Université Libre de Bruxelles (Brussels, Belgium) and the Royal College of Surgeons in Ireland with Beaumont Hospital (Dublin, Ireland).
All patients were of European ancestry as determined by self-report and genetic-marker analysis. Cases were separated according to the causal drug and then phenotyped into distinct hypersensitivity states of MPE, HSS and SJS based on the extent and severity of their ADR and using disease criteria, as previously reported [14]. In total, there were $46 \mathrm{ADR}$ cases relating to LTG, of which 42 were MPE cases, three were HSS and one was SJS. Of the 44 PHTinduced ADR cases, 40 were MPE cases and four were HSS cases. There were four patients who experienced MPE with both drugs on separate occasions and were thus included in each set of analyses. Given the low numbers of HSS and SJS patients and the evidence that a common marker may exist for all phenotypes (as evidence would suggest for $H L A-A^{*} 3101$ and CBZ-induced cADRs) we analyzed all cases together as drug-specific cADR groups.

\section{Control subjects}

The control group consisted of a homogeneous subgroup of 1296 subjects from the 1958 British birth cohort that were selected by principal component analysis of GWAS. Controls were unscreened for AED-related ADRs.

\section{Genome-wide analysis}

Genome-wide data across $>600,000$ SNPs were available for 34 LTG and 42 PHT cases and all 1296 population control subjects. Genotyping for all cases was performed with the Illumina ${ }^{\circledR}$ Human610-610K Quad platform at the Duke University Center for Human Genome Variation (NC, USA). We removed subjects with a genotyping failure rate below 95\%, SNPs with low minor allele frequency $(<2 \%)$, those failing the Hardy-Weinberg equilibrium and any that had missing genotype information in $>10 \%$ of all subjects.

Table 1. Distribution of HLA-A*3101 among lamotrigine and phenytoin case subjects compared with population control subjects.

\begin{tabular}{|c|c|c|c|}
\hline$H L A-A * 3101$ & Lamotrigine-cADR cases & Phenytoin-cADR cases & Controls \\
\hline Carriers (n; \%) & $2(4.35 \%)$ & $2(4.55 \%)$ & $72(5.56 \%)$ \\
\hline Noncarriers (n; \%) & $44(95.65 \%)$ & $42(95.45 \%)$ & $1224(94.44 \%)$ \\
\hline Total subjects (n) & 46 & 44 & 1296 \\
\hline$p$-value & 0.698 & 0.746 & \\
\hline Odds ratio $(95 \% \mathrm{Cl})$ & $0.756(0.183-3.129)$ & $0.791(0.191-3.277)$ & \\
\hline $\begin{array}{l}\text { Relative risk for } 80 \% \\
\text { power }\end{array}$ & 2.204 & 2.229 & \\
\hline
\end{tabular}




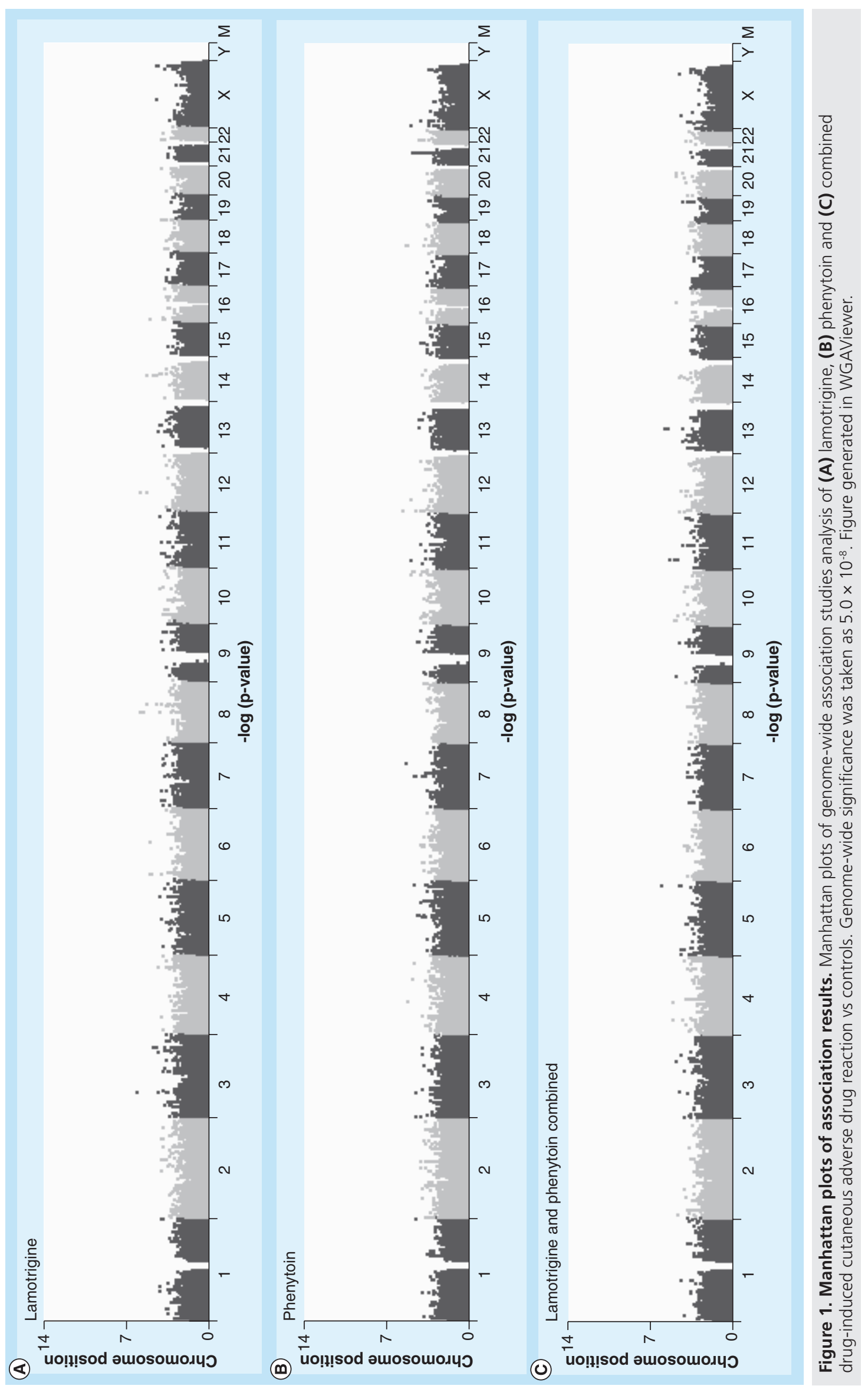




\section{Imputation of $H L A$ alleles}

We imputed classic $H L A$ alleles for all cases and controls using MACH 1.0 [17]. This method has been validated as an accurate method for imputing HLA subtypes in our cohort as described previously [14]. The HLA-typed 1958 British birth cohort constituted the reference population for imputation. For those samples for which GWAS data were not available we used PCR with sequence-specific primers to determine the presence of $H L A-A^{*} 3101$. The PCR conditions were as follows: the forward primer sequence

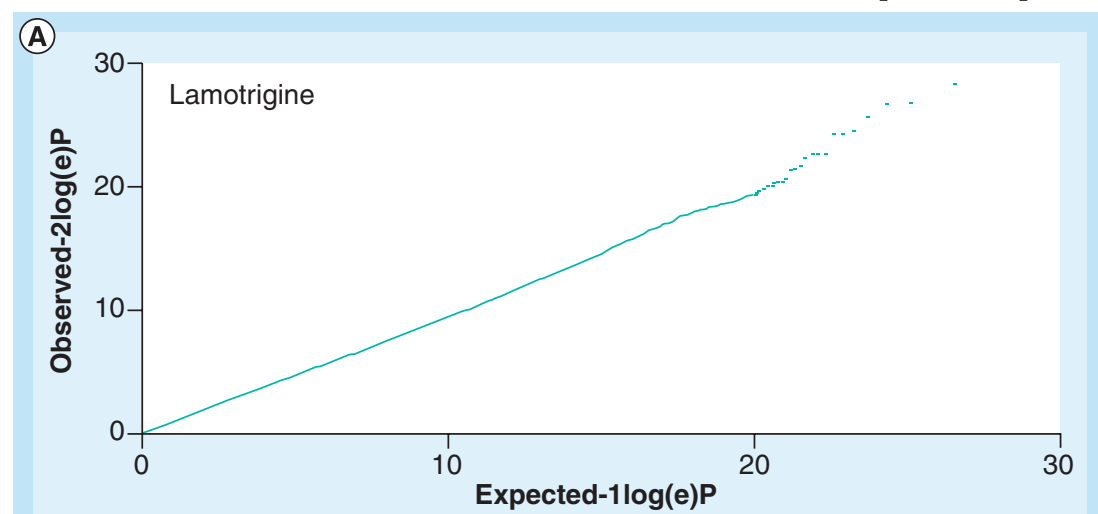

(B)

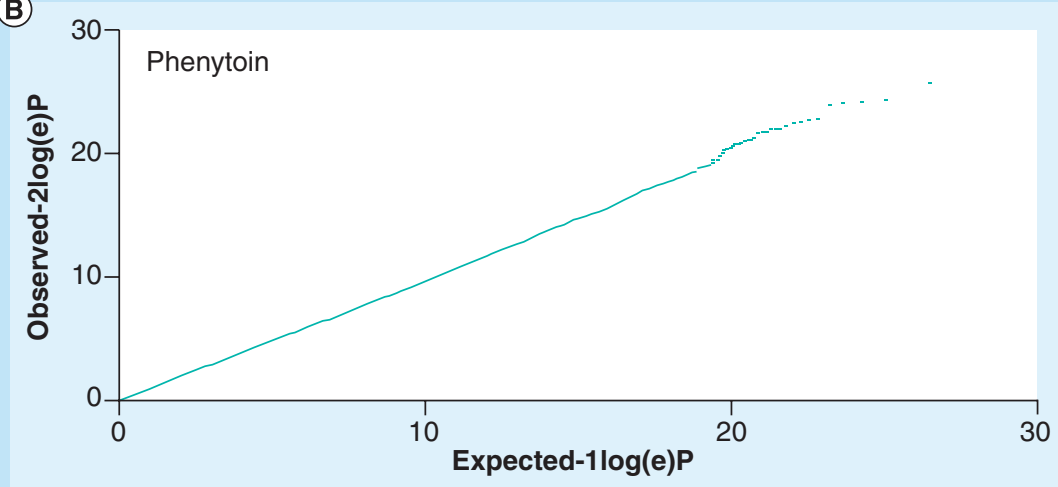

(C)

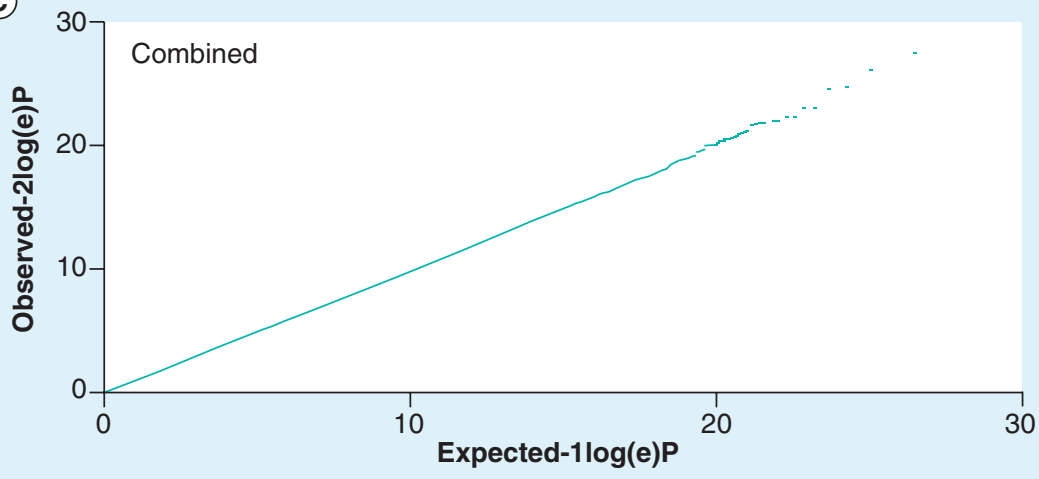

Figure 2. Quantile-quantile plot of the association results. Quantile-quantile plots of $p$-values from genome-wide association studies of $(\mathbf{A})$ lamotrigine, (B) phenytoin and (C) total drug-induced cutaneous adverse drug reactions vs controls calculated using logistic regression and including significant EIGENSTRAT axes as covariates. The expected chi distribution is represented along the $x$-axis, while the observed chi distribution is represented along the $y$-axis. Figure generated in WGAViewer. was 5'-GATAGAGCAGGAGAGGCCT-3', reverse primer sequence was 5'-AGCGCAGGTCCTCGTTCAA-3' and the cycling parameters were $95^{\circ} \mathrm{C}$ for $3 \mathrm{~min}$, five cycles of $95^{\circ} \mathrm{C}$ for $15 \mathrm{~s}, 70^{\circ} \mathrm{C}$ for $15 \mathrm{~s}$ and $72^{\circ} \mathrm{C}$ for $30 \mathrm{~s}$, two cycles of $95^{\circ} \mathrm{C}$ for $15 \mathrm{~s}, 65^{\circ} \mathrm{C}$ for $15 \mathrm{~s}$ and $72^{\circ} \mathrm{C}$ for $30 \mathrm{~s}$, four cycles of $95^{\circ} \mathrm{C}$ for $15 \mathrm{~s}, 55^{\circ} \mathrm{C}$ for $1 \mathrm{~min}$ and $72^{\circ} \mathrm{C}$ for $2 \mathrm{~min}$, finally $72^{\circ} \mathrm{C}$ for $7 \mathrm{~min}$.

\section{Statistical analysis}

Statistical analyses were performed using PLINK (version 1.05) [18] and EIGENSTRAT [19] software packages. For the GWAS, we applied logistic regression (using an additive model) to each of the cADR case groups in turn versus the 1958 British birth cohort controls. We adjusted for population stratification by including significant principal components (generated using EIGENSTRAT) as covariates in the logistic-regression model and used a p-value of less than $5.0 \times 10^{-8}$ as a threshold for genomewide significance. Surplementary Figure $\mathbf{S 1}$ (see online, www.futuremedicine.com/doi/suppl/10.2217/ PGS.11.165) for a plot of the first and second principal components between cases and controls. Post-GWAS annotation analysis was performed with WGAViewer [20]. We calculated study power using the program Power for Genetic Association Analyses [21]. We estimated the relative risk (RR) required for $80 \%$ power to detect an allelic association (under an additive disease model) with the following parameters: a prevalence of $10 \%$ of the ADR in the population, a minor allele frequency of $5 \%$, an experimentwide $\alpha$ level of 0.05 and the assumption that a genetic marker is causal or in complete linkage with the causal variant.

\section{Results}

\section{HLA-A*3101 as a predictor of LTG- and $\mathrm{PHT}$-induced CADRs}

TABLE 1 illustrates the results of $H L A-A^{*} 3101$ genotyping in LTG- and PHT-induced cADR cases and population controls. The allele does not appear to be enriched in any of the cADR patient groups. For each of the analyses considered, our power calculations indicate that we had reasonable power to detect effect sizes of clinical relevance $(\mathrm{RR}>2)$.

\section{GWAS results}

We next conducted a GWAS for each of the drug-specific cADR groups as well as a total combined case cohort. The results of the logistic regression analyses are depicted as Manhattan and quantile-quantile plots in 
Figures 1 \& 2. The ten most significant markers from the logistic regression analyses are listed in Supplementary Tables S1-S3. We did not detect any variants satisfying our threshold for genomewide significance ( $\mathrm{p}$-value of $5.0 \times 10^{-8}$ ), nor did we observe any significant deviations from the expected distribution in quantile-quantile plots (results shown in Figures 1 \& 2). From power calculations (Figure 3) we estimated that we had $80 \%$ power to detect a variant with a minor allele frequency $>10 \%$ and a RR $>2.5$ when considering all cases together. We feel this RR is within the realm of clinical relevance.

\section{Discussion}

The results presented here suggest that HLA$A^{*} 3101$ is not a clinically relevant predictor of cADRs caused by LTG and PHT in populations of European descent.

It has been shown in previous studies by ourselves and others that $H L A-A^{*} 3101$ is a strong predictor for the clinical spectrum of CBZ-induced hypersensitivity and we reported an odds ratio of 8.33 for $\mathrm{CBZ}$-induced MPE for a population of European descent, while the corresponding value for a population of Japanese descent was 10.8 [15].

We calculated that the current study had $80 \%$ power to detect a RR of 3.27 for cADRs based on a frequency of $5 \%$ for $H L A-A^{*} 3101$, which gives this study ample power to detect an effect size similar to that reported previously for CBZinduced MPE. If $H L A-A^{*} 3101$ is a predictor for either LTG- or PHT-induced cADRs, we were well powered to detect it. Of the 44 cases of PHT-induced cADRs, only two were positive for $H L A-A^{*} 3101$. Similarly, only two of the 46 cases of LTG-induced cADRs were positive for the allele. Incidentally, one of the subjects positive for $H L A-A^{*} 3101$ in the LTG case group had a historical report of a rash with $\mathrm{CBZ}$.

Although we included HSS and SJS, the overwhelming majority of cases were of the MPE type. Given the small number of HSS and SJS cases, we pooled all cases into a single group of hypersensitivity ADR cases per drug for a better powered genome-wide analysis. We chose to analyze all cases together as cADR groups as we did not have enough subjects to run a powered GWAS for HSS or SJS alone. The GWAS results remain negative when the HSS and SJS cases are removed. Further studies of PHT- and LTGinduced HSS and SJS in larger patient cohorts is warranted, especially given that the genetic predictors (e.g., $H L A-B^{*} 1502$ ) can be selective for a given hypersensitivity reaction $[5,13,22]$, and

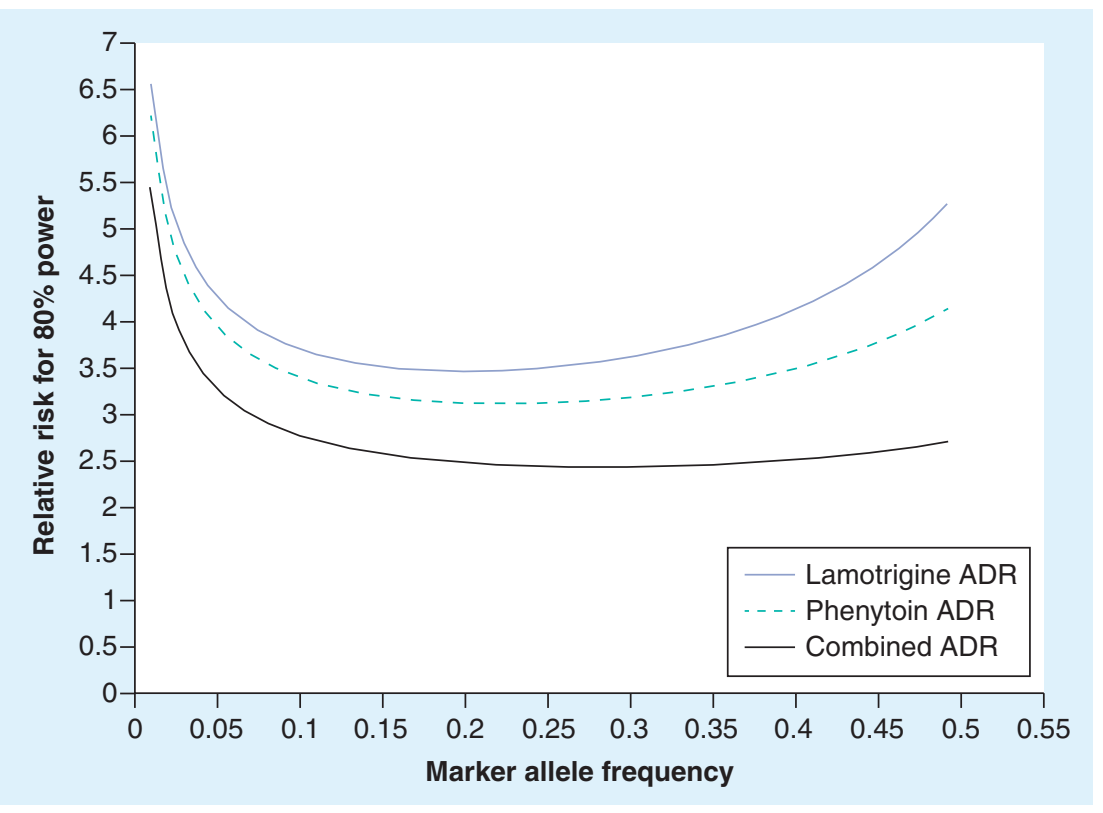

Figure 3. Power calculation for genome-wide association results.

The three analyses are represented with lamotrigine, phenytoin and combined drug cutaneous ADR groups with 1296 controls. Taking all cases together, we estimated we had $80 \%$ power to detect a variant with a minor allele frequency $>10 \%$ and a relative risk $>2.5$.

ADR: Adverse drug reaction.

the effect size correlates with the severity of the phenotype across the hypersensitivity spectrum (as appears to be the case for $H L A-A^{*} 3101$ and CBZ [14,16]).

Our GWAS results are not suggestive of a common, clinically relevant predictor of LTG- or PHT-induced cADRs in a European population. None of the variants tested satisfied the GWAS significance threshold, nor was there any clear biological candidate from within the pool of the most significant SNPs (Supplementary Tables S1-S3). Our study was powered to detect a variant of minor allele frequency $>10 \%$ carrying a clinically relevant genetic effect $(\mathrm{RR}>2.5)$. We recognize that sample size is a major limitation of our study. This has limited our analysis to effect sizes in the realm of clinical relevance (for cADRs) and has prevented us from studying SJS/TEN specifically. We also cannot rule out genetic variants conveying smaller effects $(R R<2.5)$ or rare variants of small or large effect, which were not captured by our GWAS chip.

With the increased availability of wholeexome and whole-genome sequencing, it should become possible and more affordable to identify rare variants not captured through GWAS. Discovering these genetic markers would further the understanding of the functional role of HLA in drug response, as well as helping clinicians screen patients susceptible to these ADRs. This study mainly focused on patients 
with the less severe MPE hypersensitivity reaction; however, there is a clear motivation to investigate whether $H L A-A^{*} 3101$ extends itself as a genetic marker for HSS and SJS/TEN caused by LTG and PHT.

\section{Conclusion}

$H L A-A^{*} 3101$ does not appear to be a genetic marker for LTG- and PHT-induced MPE reactions in Europeans. The predictive application of $H L A-A^{*} 3101$ as a clinically relevant genetic marker for cADRs in Europeans appears to be specific to CBZ.

Financial \& competing interests disclosure
This work was supported by grants from the Medical
Research Council (G0400126), the Wellcome Trust
(084730), UCLH CRDC (F136), the National Institute
for Health Research (08-08-SCC), Brainwave - the Irish
Epilepsy Association/the Medical Research Charities Group
of Ireland/Health Research Board award (2009/001), the
National Society for Epilepsy, the Fonds National de la
Recherche Scientifique and the Fonds Erasme pour la
Recherche Médicale, UniversitéLibre de Bruxelles (Belgium)
andsupported, in part, by Award Number RC2NSO70344 from the National Institute of Neurological Disorders and Stroke. M McCormack was supported by a Health Research Board of Ireland's Translational Research Scholars award. The authors acknowledge use of genotype data from the 1958 British birth cohort DNA collection, funded by the Medical Research Council grant G0000934 and the Wellcome Trust grant 068545/Z/02. The collection of the Belgian patients was supported by the Fonds National de la Recherche Scientifique grant number FC 63574/3.4.620.06 F, and the Fonds Erasme pour la Recherche Médicale, Université Libre de Bruxelles (Belgium). The authors have no other relevant affiliations or financial involvement with any organization or entity with a financial interest in or financial conflict with the subject matter or materials discussed in the manuscript apart from those disclosed.

No writing assistance was utilized in the production of this manuscript.

\section{Ethical conduct of research}

The authors state that they have obtained appropriate institutional review board approval or have followed the principles outlined in the Declaration of Helsinki for all human or animal experimental investigations. In addition, for investigations involving human subjects, informed consent has been obtained from the participants involved.

\section{Executive summary}

\section{Background}

- HLA-B*1502 is a genetic predictor for Stevens-Johnson syndrome/toxic epidermal necrolysis caused by carbamazepine (CBZ) and phenytoin (PHT) among Han Chinese and south-east Asian populations, while HLA-A*3101 has recently been shown to associate with the full spectrum of CBZ-induced cutaneous adverse drug reactions (CADRs; maculopapular eruption, hypersensitivity syndrome and Stevens-Johnson syndrome/toxic epidermal necrolysis) in European and Japanese populations.

- With this knowledge we investigated whether HLA-A*3101 or any other common genetic marker was associated with lamotrigine(LTG) or PHT-induced CADRs.

\section{Results}

- There was no association between HLA-A*3101 and LTG- or PHT-induced CADRs.

- We did not find a significant common genetic variant from our genome-wide analyses.

\section{Conclusion}

- We have shown that the association with HLA-A*3101 does not extend to LTG- or PHT-induced cADRs. We also did not detect any other significant common variants of association with LTG- or PHT-induced CADRs from genome-wide association studies.

- HLA-A*3101 thus appears to be a specific marker for CBZ-induced CADRs.

\section{References}

Papers of special note have been highlighted as:

- of interest

" of considerable interest

1 Arif H, Buchsbaum R, Weintraub D et al. Comparison and predictors of rash associated with 15 antiepileptic drugs. Neurology 68(20), 1701-1709 (2007).

2 Chung WH, Hung SI, Hong HS et al. Medical genetics: a marker for StevensJohnson syndrome. Nature 428(6982), 486 (2004).

- - The first study to report the association between $H L A-B^{*} 1502$ and Stevens-Johnson syndrome/toxic epidermal necrolysis. Based on the results of this study and further replications, the US FDA approved a label change to carbamazepine.

3 Hung SI, Chung WH, Liu ZS et al. Common risk allele in aromatic antiepileptic-drug induced Stevens-Johnson syndrome and toxic epidermal necrolysis in Han Chinese. Pharmacogenomics 11(3), 349-356 (2010).

4 Ding WY, Lee CK, Choon SE. Cutaneous adverse drug reactions seen in a tertiary hospital in Johor, Malaysia. Int. J. Dermatol. 49(7), 834-841.

5 Locharernkul C, Loplumlert J, Limotai C et al. Carbamazepine and phenytoin induced Stevens-Johnson syndrome is associated with $H L A-B^{*} 1502$ allele in Thai population. Epilepsia 49(12), 2087-2091 (2008).
6 Mehta TY, Prajapati LM, Mittal B et al. Association of $H L A-B^{*} 1502$ allele and carbamazepine-induced Stevens-Johnson syndrome among Indians. Indian J. Dermatol. Venereol. Leprol. 75(6), 579-582 (2009).

7 Shi YW, Min FL, Liu XR et al. Hla-B alleles and lamotrigine-induced cutaneous adverse drug reactions in the Han Chinese population. Basic Clin. Pharmacol. Toxicol. 109(1), 42-46 (2011).

8 An DM, Wu XT, Hu FY, Yan B, Stefan H, Zhou D. Association study of lamotrigineinduced cutaneous adverse reactions and $H L A-B^{*} 1502$ in a Han Chinese population. Epilepsy Res. 92(2-3), 226-230 (2010). 
9 Hu FY, Wu XT, An DM, Yan B, Stefan H, Zhou D. Pilot association study of oxcarbazepine-induced mild cutaneous adverse reactions with $H L A-B^{*} 1502$ allele in Chinese Han population. Seizure 20(2), 160-162 (2011).

10 Alfirevic A, Jorgensen AL, Williamson PR, Chadwick DW, Park BK, Pirmohamed M. $H L A-B$ locus in Caucasian patients with carbamazepine hypersensitivity. Pharmacogenomics 7(6), 813-818 (2006).

11 Lonjou C, Thomas L, Borot $\mathrm{N}$ et al. A marker for Stevens-Johnson syndrome ethnicity matters. Pharmacogenomics J. 6(4), 265-268 (2006).

12 Gonzalez-Galarza FF, Christmas S, Middleton D, Jones AR. Allele frequency net: a database and online repository for immune gene frequencies in worldwide populations. Nucleic Acids Res. 39(Database issue), D913-D919 (2011).

13 Hung SI, Chung WH, Jee SH et al. Genetic susceptibility to carbamazepine-induced cutaneous adverse drug reactions. Pharmacogenet. Genomics 16(4), 297-306 (2006).

14 Mccormack M, Alfirevic A, Bourgeois S et al. $H L A-A^{*} 3101$ and carbamazepine-induced hypersensitivity reactions in Europeans. N. Engl. J. Med. 364(12), 1134-1143 (2011).

- - The first paper to report an association between $H L A-A^{*} 3101$ and the spectrum of carbamazepine-induced cutaneous adverse drug reactions in Europeans.

15 Ozeki T, Mushiroda T, Yowang A et al. Genome-wide association study identifies $H L A-A^{*} 3101$ allele as a genetic risk factor for carbamazepine-induced cutaneous adverse drug reactions in Japanese population. Hum. Mol. Genet. 20(5), 1034-1041 (2010).

- Identified $H L A-A^{*} 3101$ as a marker for carbamazepine-induced cutaneous adverse drug reactions in a Japanese population, extending the global range to which this marker may apply.

16 Kim SH, Lee KW, Song WJ et al. Carbamazepine-induced severe cutaneous adverse reactions and $H L A$ genotypes in Koreans. Epilepsy Res. 97(1-2), 190-197 (2011).

17 Li Y, Willer CJ, Ding J, Scheet P, Abecasis GR. $\mathrm{MaCH}$ : using sequence and genotype data to estimate haplotypes and unobserved genotypes. Genet. Epidemiol. 34(8), 816-834. (2010).
18 Purcell S, Neale B, Todd-Brown K et al. PLINK: a tool set for whole-genome association and population-based linkage analyses. Am. J. Hum. Genet. 81(3), 559-575 (2007).

19 Price AL, Patterson NJ, Plenge RM, Weinblatt ME, Shadick NA, Reich D. Principal components analysis corrects for stratification in genome-wide association studies. Nat. Genet. 38(8), 904-909 (2006).

20 Ge D, Zhang K, Need AC et al. WGAViewer: software for genomic annotation of whole genome association studies. Genome Res. 18(4), 640-643 (2008).

21 Menashe I, Rosenberg PS, Chen BE. PGA: power calculator for case-control genetic association analyses. BMC Genet. 9, 36 (2008).

22 Man CB, Kwan P, Baum L et al. Association between $H L A-B^{*} 1502$ allele and antiepileptic drug-induced cutaneous reactions in Han Chinese. Epilepsia 48(5), 1015-1018 (2007).

23 Solberg OD, Mack SJ, Lancaster AK et al. Balancing selection and heterogeneity across the classical human leukocyte antigen loci: a meta-analytic review of 497 population studies. Hum. Immunol. 69 (7), 443-464 (2008). 